\title{
Electromagnetic Interactions in Nuclear and Hadron Physics
}


This page is intentionally left blank

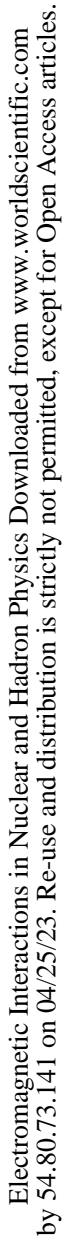




\section{Proceedings of the International Symposium}

\section{Electromagnetic Interactions in Nuclear and Hadron Physics}

Osaka, Japan 4-7 December 2001

editors

\section{Mamoru Fujiwara Tatsushi Shima}

Research Center for Nuclear Physics, Osaka University, Japan 


\section{Published by}

World Scientific Publishing Co. Pte. Ltd.

P O Box 128, Farrer Road, Singapore 912805

USA office: Suite 1B, 1060 Main Street, River Edge, NJ 07661

UK office: 57 Shelton Street, Covent Garden, London WC2H 9HE

\section{British Library Cataloguing-in-Publication Data}

A catalogue record for this book is available from the British Library.

\section{ELECTROMAGNETIC INTERACTIONS IN NUCLEAR AND HADRON PHYSICS Proceedings of the International Symposium}

Copyright $(\mathcal{O} 2002$ by World Scientific Publishing Co. Pte. Ltd.

All rights reserved. This book, or parts thereof, may not be reproduced in any form or by any means, electronic or mechanical, including photocopying, recording or any information storage and retrieval system now known or to be invented, without written permission from the Publisher.

For photocopying of material in this volume, please pay a copying fee through the Copyright Clearance Center, Inc., 222 Rosewood Drive, Danvers, MA 01923, USA. In this case permission to photocopy is not required from the publisher.

ISBN $\quad 981-238-044-2$ 


\title{
International Symposium on
}

\section{Electromagnetic Interactions in Nuclear and Hadron Physics (EMI2001)}

RCNP, Osaka, Japan December 4-7, 2001

\section{International advisorv committee}

Sam M. Austin (NSCL), I.T. Cheon (Yonsei), K. de Jager (JLab),

S. Gales (Orsay), M.N. Harakeh (KVI), K. Hicks (Ohio),

H. Horiuchi (Kyoto), H. Kamitsubo (SPring-8),

T.-S.H. Lee (Argonne), B. Mecking (JLab), Y. Nagai (RCNP),

S. Nagamiya (KEK), A. Sandorfi (BNL), C. Schaerf (Rome), H. Stroeher (Jeulich), Z. Suikowski (Swierk), I. Tanihata (RIKEN), H. Toki (RCNP), M. Urin (Moscow)

\section{Organizing committee}

M. Fujiwara (RCNP/JAERI ; Chairperson), T. Nakano (RCNP), H. Shimizu (Yamagata), H. Utsunomiya (Konan), T. Kishimoto (Osaka), T. Sato (Osaka), T. Morii (Kobe), J. Kasagi (Tohoku), Y. Ohashi (SRing-8), S. Date (Spring-8), H. Sagawa (Aizu), T. Shima (RCNP ; Scientific secretary), K. Takahisa (RCNP)

\author{
Host Institute \\ Research Center for Nuclear Physics (RCNP) \\ Osaka University
}




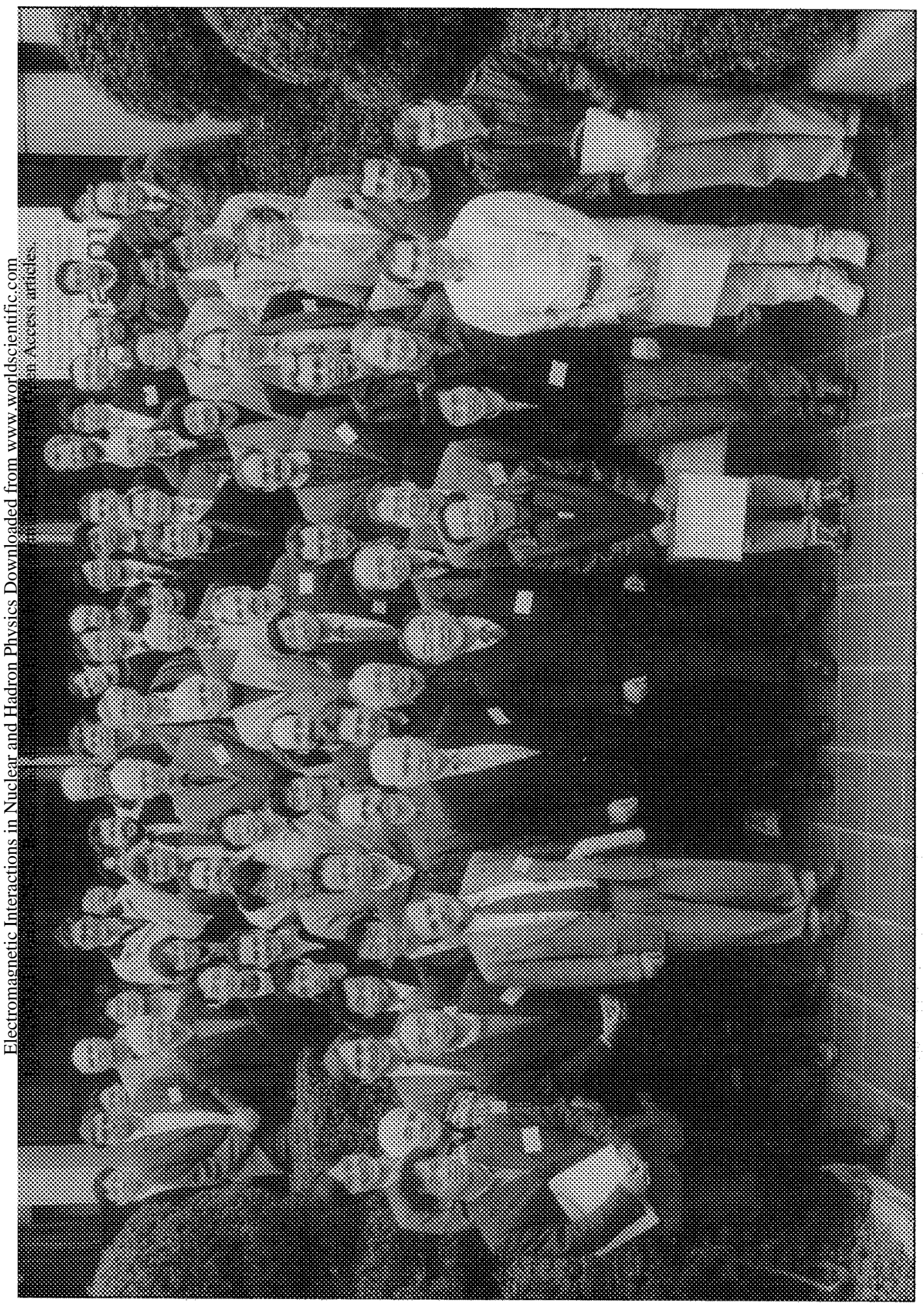




\section{PREFACE}

The International Symposium on Electromagnetic Interactions in Nuclear and Hadron Physics (EMI2001) was held at Osaka University, Japan from December 4 th to 7 th, 2001. The symposium is a topical one to discuss the fundamental problems in nuclear and hadron physics developed with "electromagnetic probes". When a decision was made nearly two years ago to organize this symposium, several movements towards new developments of physics with electromagnetic probes were expected to be common in the world. Actually, interesting and hot results from many laboratories in the world have been presented and discussed in the symposium.

This symposium is supported by Ministry of Education, Culture, Sport, Science and Technology (Monbu-Kagaku-shou) under COE (Center of Excellence) Program, and is hosted by the Research Center for Nuclear Physics (RCNP). The symposium belongs to a series of international meetings at RCNP. This time, we would like to organize the symposium to discuss nuclear and hadron physics with emphasis on real and virtual photon interactions. It is timely to discuss the subjects mentioned above since we expect to have some preliminary results from the LEPS (Laser-Electron-Photon Spectrometer) facility at SPring-8 using the photon beam with an energy of $1.5-2.4 \mathrm{GeV}$, and since other new technical developments will be delivered for future experiments.

Subjects discussed in the symposium are

1. Meson and hadron productions by real and virtual photon interaction with nucleons and nuclei

2. Astrophysics studies via photoreactions and hadron reactions

3. New technologies for the electromagnetic (E.M.) probes and the detector development

4. Nuclear structure studied with E.M. probes

5. Fundamental symmetries with E.M. probes and related problems

The symposium is intended also for celebrating the 30 years anniversary of RCNP, Osaka University. The ceremony and reception of the 30 years RCNP anniversary is held on December 3rd, 2001, before the symposium.

In addition to invited talks, we arranged a poster session and a special session where the students working at RCNP presented their latest results for the attendant experienced scientists. The number of the participants was 
viii

about 130 including 80 scientists from outside Japan. We could have lively discussions with ample time.

On behalf of the organizing committee, we thank many students and young researchers of RCNP for their great help during the symposium. We appreciate the RCNP official staff for their considerable help to make the present symposium successful. They include A. Nagasawa, S. Yoshida, Y. Inui, M. Wada, S. Kinjo, T. Murakami, A. Nakazawa, Y. Ogawa, S. Hyodo, Y. Ito, C. Sano, M. Nishimoto and M. Matsuura.

We would like to acknowledge the generous offer of the Welding Research Institute of Osaka University for allowing the use of the Arata Memorial Hall as the main meeting hall of the EMI2001 symposium.

Finally, we thank the members of the International Advisory Committee and the Organizing Committee for their sound advice which helped us to prepare the symposium. One of our scientific goals was to publish this EMI2001 Proceedings in a relatively short time after the symposium that is important to encourage the subsequent further scientific work. We wish to thank all the speakers for submitting their contribution papers.

Editors: Mamoru Fujiwara Tatsushi Shima 


\section{CONTENTS}

Preface

Nucleon Electromagnetic Form Factors

K. de Jager

Search for New Baryon Resonances

B. Saghai and $Z$. $L i$

Overview of Laser-Electron Photon Facility at SPring-8 T. Nakano

Weak Nucleon Form Factors

P. A. Souder

Parity Violating Electron-Proton Scattering Experiments

W. T. H. van Oers

Parity Violation Experiment at Mainz

$J$. van de Wiele and M. Morlet

Parity Violating Measurements of Neutron Densities: Implications for Neutron Stars

C. J. Horowitz and J. Piekarewicz

High Energy Approaches to Low Energy Phenomena in Astrophysics

S. M. Austin

Nuclear Astrophysics Experiments with Laser-Electron $\mathrm{MeV}$ Photons

H. Utsunomiya, S. Goko, H. Akimune, K. Yamasaki,

T. Yamagata, M. Ohta, H. Ohgaki, H. Toyokawa, Y.-W. Lui,

T. Hayakawa, T Shizuma, K. Sumiyoshi and T. Kajino

Open Questions in Stellar Helium Burning Studied with Photons

M. Gai

Photo-Nuclear Reactions in the Big-Bang and Supernovae

T. Kajino, M. Orito, K. Otsuki and M. Terasawa 
The Fundamental $\gamma N \rightarrow \pi N$ Processes at JLAB Energies H. Gao

The New Crystal Ball Experimental Program

W. J. Briscoe

The First Pion Photoproduction Results from Polarized HD at LEGS C. S. Whisnant, K. Ardashev, V. Bellini, M. Blecher, C. Cacace, A. Caracappa, A. Cichocki, C. Commeaux, I. Danchev,

A. D'Angelo, J. P. Didelez, R. Deininger, C. Gibson, K. Hicks,

S. Hoblit, A. Honig, T. Kageya, M. Khandaker, O. Kistner,

A. Kuczewski, F. Lincoln, R. Lindgren, A. Lehmann, M. Lowry,

M. Lucas, J. Mahon, H. Meyer, L. Miceli, D. Moriccianni,

B. M. Preedom, B. Norum, T. Saitoh, A. M. Sandorfi,

C. Shaerf, D. J. Tedeschi, C. Thorn, K. Wang and X. Wei

Neutral Pion Production from Deuterium at the LEGS Facility

K. H. Hicks, K. Ardashev, M. Blecher, A. Caracappa,

A. Cichocki, C. Commeaux, A. D'Angelo, J.-P. Didilez,

R. Deininger, S. Hoblit, M. Khandaker, O. Kistner,

A. Kuczewski, F. Lincoln, R. Lindgren, A. Lehmann, M. Lowry,

M. Lucas, H. Meyer, L. Miceli, A. Opper, B. M. Preedom,

B. Norum, A. M. Sandorfi, C. Schaerf, H. Ströher, C. E. Thorn,

J. Tonnison, K. Wang, X. Wei, C. S. Whisnant and D. Willits

Transition Properties of Low-Lying Resonances in a Relativistic

Quark Model with a Meson Cloud Effect

Y. B. Dong, A. Faessler and K. Shimizu

Studies of the Dilepton Emission from Nucleon-Nucleon Interactions J. C. S. Bacelar

Pion Electroproduction off ${ }^{3} \mathrm{He}$ and Self Energies of the Pion and the $\Delta$ Isobar in the Medium

A. Richter

The Deuteron Electromagnetic Form Factors

G. G. Petratos

Searching for Quarks in the Deuteron

R. Gilman 
Photoreactions in Nuclear Astrophysics

P. Mohr, M. Babilon, W. Bayer, D. Galaviz, T. Hartmann,

C. Hutter, K. Sonnabend, K. Vogt, S. Volz and A. Zilges

Weak Interaction, Giant Resonances and Nuclear Astrophysics

K. Langanke and G. Martinez-Pinedo

The HI $\gamma$ S Facility -- A Free-Electron Laser Generated Gamma-Ray Beam for Nuclear Physics

H. R. Weller

Parametrization of Parton Distribution Functions in Nuclei

S. Kumano and M.-A. Nakamura

Roper Electroproduction Amplitudes in a Chiral Confinement Model

M. Fiolhais, P. Alberto, J. Marques and B. Golli

$\mathrm{S}_{11}$ (1535) Resonance in Nuclear Medium Observed with the $(\gamma, \eta)$ Reactions

H. Yamazaki, T. Kinoshita, K. Hirota, K. Kino, T. Nakabayashi,

T. Katsuyama, A. Katoh, T. Terasawa, H. Shimizu, J. Kasagi,

T. Takahashi, H. Kanda, K. Maeda, Y. Tajima, H. Y. Yoshida,

T. Noma, Y. Aruga, A. Iijima, Y. Ito, T. Fujinoya, T. Yorita and $O$. Konno

Final State Interactions in $\omega$ Photoproduction Near Threshold

Y. Oh and T.-S. H. Lee

The $Q^{2}$ Evolution of the GDH Sum Rule (on ${ }^{3} \mathrm{He}$ and the Neutron)

G. D. Cates

Detailed Study of the ${ }^{3} \mathrm{He}$ Nuclei through Response Function Separations at High Momentum Transfer

D. W. Higinbotham

Final State Interaction in ${ }^{4} \mathrm{He}\left(e, e^{\prime} p\right){ }^{3} \mathrm{H}$ Reaction: Study of Finite Formation Time Effects

H. Morita, C. Ciofi degli Atti, D. Treleani and M. A. Braun 
Simultaneous Measurement of the Two-Body Photodisintegration of ${ }^{3} \mathrm{H}$ and ${ }^{3} \mathrm{He}$

G. V. O'Rielly

Nuclear Medium Effects in Hadron Leptoproduction

N. Bianchi

Quasifree Processes from Nuclei: Meson Photoproduction and Electron Scattering

L. J. Abu-Raddad and J. Piekarewicz

Quasielastic and $\Delta$ Excitation in Electron Scattering

K. S. Kim, S. W. Hong, B. T. Kim and T. Udagawa

Kaon Photo- and Electroproduction on the Deuteron with Beam and Recoil Polarizations

K. Miyagawa, T. Mart, C. Bennhold and W. Glöckle

Electroproduction of Strange Nuclei

E. V. Hungerford

Photoproducion of the $\phi(1020)$ Near Threshold in CLAS

D. J. Tedeschi for the CLAS Collab.

$K^{+}$Photoproduction at LEPS/SPring- 8

R. G. T. Zegers, Y. Asano, N. Muramatsu, M. Fujiwara,

H. Fujimura, T. Hotta, H. Kohri, T. Matsumura, N. Matsuoka,

T. Mibe, M. Morita, T. Nakano, K. Yonehara, T. Yorita,

S. Daté, N. Kumagai, Y. Ohashi, H. Ookuma, W. C. Chang,

D. S. Oshuev, C. W. Wang, S. C. Wang, K. Imai, T. Ishikawa,

M. Miyabe, N. Niyama, M. Yosoi, H. Kawai, T. Ooba,

Y. Shiino, D. S. Ahn, J. K. Ahn, S. Makino, T. Iwata,

Y. Miyachi, A. Wakai, M. Nomachi, A. Sakaguchi, Y. Sugaya,

M. Sumihama, H. Akimune, C. Rangacharyulu, K. Hicks,

H. Shimizu, P. Shagin, H. C. Bhang and Z. Y. Kim

Polarization Observables in Kaon Electroproduction with CLAS at Jefferson Laboratory

D. S. Carman 
Can the Scalar Mesons $a_{0} / f_{0}(980)$ be Described by $K+\bar{K}$ ?

R. T. Jones

Meson Photoproduction at GRAAL

O. Bartalini, V. Bellini, J. P. Bocquet, M. Capogni, M. Castoldi,

A. D'Angelo, Annelisa D'Angelo, J. P. Didelez, R. di Salvo,

A. Fantini, G. Gervino, F. Ghio, B. Girolami, M. Guidal,

E. Hourany, I. Kilvington, R. Kunne, V. Kuznetsov, A. Lapik,

P. Levi Sandri, A. Lleres, D. Moricciani, V. Nedorezov,

L. Nicoletti, D. Rebreyend, F. Renard, N. V. Rudnev,

C. Schaerf, M. L. Sperduto, M. C. Sutera, A. Turinge

and A. Zucchiatti

Giant Resonances in Nuclei Near and Far from $\beta$-Stability Line

H. Sagawa

Indirect Measurements of the ${ }^{7} \mathrm{Be}(p, \gamma)^{8} \mathrm{~B}$ Reaction

T. Motobayashi

Search for an Orbital Magnetic Quadrupole Twist Mode in Nuclei with Electron Scattering at $180^{\circ}$

P. von Neumann-Cosel

Spin-Isospin Interaction and Properties in Stable and Exotic Nuclei T. Otsuka, R. Fujimoto, Y. Utsuno, B. A. Brown, M. Honma and T. Mizusaki

Photonuclear Reactions of Light Nuclei and Few-Body Problems T. Shima, Y. Nagai, S. Naito, K. Tamura, H. Ohgaki, T. Kii and H. Toyokawa

Determination of $S_{17}$ Based on CDCC Analyses for ${ }^{7} \mathrm{Be}(d, n)^{8} \mathrm{~B}$

K. Ogata, M. Yahiro, Y. Iseri and M. Kamimura

E2 and M1 Transitions Among Triaxially Superdeformed Bands in Lu Isotopes

K. Sugawara-Tanabe and K. Tanabe

On Description of Photonuclear Reactions Accompanied by

Excitation of the Giant Dipole Resonance

V. A. Rodin and M. H. Urin 
xiv

Isoscalar and Isovector, Spin and Orbital Contributions in M1 Transitions

Y. Fujita, Y. Shimbara, T. Adachi, G. P. A. Berg, H. Fujimura, H. Fujita, I. Hamamoto, K. Hara, K. Hatanaka, F. Hofmann, J. Kamiya, T. Kawabata, Y. Kitamura, P. von Neumann-Cosel, A. Richter, Y. Shimizu, M. Uchida, K. Yamasaki, M. Yoshifuku and $M$. Yosoi

Electromagnetic Processes in Drip-Line Nuclei

T. Suzuki, T. Otsuka and R. Fujimoto

Total Photo-Absorption of Actinide Nuclei at Intermediate Energies

V. G. Nedorezov

Compton Scattering on the Proton and Light Nuclei in the

$\Delta$-Resonance Region

O. Scholten and S. Kondratyuk

Semi-Inclusive Large- $P_{T}$ Light Hadron Pair Production as a Probe of Polarized Gluons

T. Morii, Y. B. Dong and T. Yamanishi

Strange-Quark Current in the Nucleon from Lattice QCD

R. Lewis, W. Wilcox and R. M. Woloshyn

Quantum Entanglement Measurements of Two Spin 1/2 Hadrons

C. Rangacharyulu

Single and Double Spin Azimuthal Asymmetries in Semi-Inclusive Pion Electroproduction

$$
\text { K. A. Oganessyan }
$$

On Use of Quark-Hadron Duality in Photoabsorption Sum Rules

S. B. Gerasimov

Experimental Studies in Gerasimov-Drell-Hearn Sum Rule

T. Iwata

Forward Compton Scattering: Sum Rules for Parity Violating Spin Polarizabilities

L. Lukaszuk 
Generalized Parton Distributions

M. Guidal

Chiral Symmetry and Hadron Properties in Lattice QCD

A. W. Thomas

612

Determination of the Axial Coupling Constant $G_{A}$ in the Linear Representations of Chiral Symmetry

A. Hosaka, D. Jido and M. Oka

622

Study $\omega$ and $\phi$ Photoproduction in the Nucleon Isotopic

Channels

Q. Zhao

632

The Role of Low Mass Nucleon Resonances in Near Threshold $\omega$ Meson Photoproduction

A. I. Titov and T.-S. Harry Lee

Photoproduction Experiments with Polarized HD Targets

S. Bouchigny, C. Commeaux, J.-P. Didelez, G. Rouille, M. Guidal, E. Hourany, R. Kunne, M. Bassan, A. D'Angelo, R. di Salvo, A. Fantini, D. Moricciani, C. Schaerf, V. Bellini, J.-P. Bocquet, A. Lleres, D. Rebreyend, M. Castoldi, A. Zucchiatti, G. Gervino, F. Ghio, B. Girolami, O. Bartalini and P. Levi Sandri

Compton Scattering and $\pi^{+}$Photo-Production at GRAAL O. Bartalini, V. Bellini, J. P. Bocquet, M. Capogni, M. Castoldi, A. D'Angelo, Annelisa D'Angelo, J. P. Didelez, R. di Salvo, A. Fantini, G. Gervino, F. Ghio, B. Girolami, M. Guidal, E. Hourany, I. Kilvington, R. Kunne, V. Kuznetsov, A. Lapik, P. Levi Sandri, A. Lleres, D. Moricciani, V. Nedorezov, L. Nicoletti, D. Rebreyend, N. V. Rudnev, C. Schaerf, M. L. Sperduto, M. C. Sutera, A. Turinge and A. Zucchiatti

New $\left(e, e^{\prime} K^{+}\right)$Hypernuclear Spectroscopy with a High-Resolution Kaon Spectrometer (HKS)

O. Hashimoto 
Development of a Compact Photon Detector for ANKE at COSY Jülich

M. Büscher, V. Hejny, H. R. Koch, H. Machner, H. Seyfarth,

H. Ströher, M. Hoek, R. Novotny, K. Römer, J. Bacelar,

H. Löhner, A. Magiera, A. Wrońska and V. Chernychov

The Commissioning of the Hall-B Beamline of Jefferson Lab for Coherently Producing a Beam of Linearly-Polarized Photons

P. L. Cole, J. Kellie, F. J. Klein, K. Livingston, J. A. Mueller, J. C. Sanabria and D. J. Tedeschi

List of Participants 